\title{
THE EFFECT OF DYNAMICALLY UNSTABLE CHANNELING ON OFF-AXIS ION IMPLANTATION
}

\author{
M. I. Bratchenko, A. S. Bakai, S. V. Dyuldya \\ National Scientific Center "Kharkiv Institute of Physics and Technology", \\ 1, Akademichna St., UA-61108, Kharkiv, Ukraine
}

(Received June 27, 2008)

\begin{abstract}
Theoretical consideration and extensive numerical simulations are made to investigate directional effects at the implantation of heavy ions into single crystal targets. A novel molecular dynamics code MICKSER is developed for the simulations. The main goal is to solve the problem of suppression of the long-range channeling tails at off-axis implantation. It is revealed for the first time that a part of the above-barrier ions possess the specific mode of dynamically unstable directed motion, the metachanneling. It is a transient mode between the stable channeling and quasichanneling providing a reduced stopping of ions and affecting the as-implanted doping profiles. The analytical model resulted in new critical transverse energy. The critical angle of metachanneling has been proposed. They determine the upper bound of the energy losses channeling dip at low-energy ion implantation. It has been demonstrated that the minimization of channeling tails can be practically achieved when tilting a target by the critical angle of metachanneling.

Key words: ion implantation, silicon, doping profile, channeling, molecular dynamics, Monte Carlo method.
\end{abstract}

PACS number(s): 61.72.Tt, 85.40.Ry, 61.85.+p, 02.70.Ns, 02.70.Uu

\section{INTRODUCTION}

Ion implantation $[1,2]$ is a mainstream irradiation technology of the fabrication of integrated circuits (ICs) and an essential process step of the modern Complementary Metal Oxide Semiconductor (CMOS) technology platform [3]. Directed beams of low-to-medium energy $\left(10^{2} \div 10^{5} \mathrm{eV}\right)$ heavy ions are used to introduce dopants (B, P, As, In, Sb, etc.) into wafers of semiconductors ( $\mathrm{Si}$, $\mathrm{Ge}, \mathrm{GaAs}, \mathrm{SiC}$ and so on) at low temperatures and a wide range of doses $\left(10^{11} \div 10^{16}\right.$ ions $\left./ \mathrm{cm}^{-2}\right)$ in order, e. g., to adjust the threshold voltage of MOS transistors. Highenergy $(\sim \mathrm{MeV})$ high-dose $\left(\sim 10^{18} \mathrm{~cm}^{-2}\right)$ implantation is used to form heavily-doped buried insulator layers.

The advance to the nanoscale $(<50 \mathrm{~nm})$ dimensions of IC components poses a challenge for the accuracy of prediction of three-dimensional (3D) spatial distributions of dopants that is crucial for the IC manufacturing process control [3]. Implantation is very sensitive to the dopant sort, energy $E$, and dose as well as to the wafer structure, orientation and temperature. It applies yet to the "as-implanted" doping profiles formed at the primary dynamical stage of ion-atomic collisions that precedes the posterior stages of dopant redistribution at rapid thermal annealing (RTA) and irradiation induced transient enhanced diffusion (TED).

The physically based approach to the as-implanted dopant distributions calculations is the $3 \mathrm{D}$ atomistic Monte Carlo (MC) simulation of the transport of energetic ions in solid. For amorphous targets, the precise knowledge of nuclear and electronic energy losses of ions [4] is quite sufficient to predict the as-implanted dopant concentration $C(z)$ at the depth $z$ using the stateof-the-art MC codes like SRIM [5].

The Gaussian shaped $C(z) \propto \exp \left(-\frac{\left(z-R_{\mathrm{p}}\right)^{2}}{2 \delta R_{\mathrm{p}}^{2}}\right)$ deriv- ed from the ions projected range $R_{\mathrm{p}}(E)$ and straggling $\delta R_{\mathrm{p}}(E)$ are qualitatively inherent to disordered materials though the asymmetric Pearson IV function fits $C(z)$ at $z<R_{\mathrm{p}}$ more accurately.

But since typical target wafers are crystalline, the fast ions atomic collisions and stopping are strongly affected by their regular lattice structure. Channeling $[6,7]$, the steered motion of energetic ions governed by averaged continuum potentials of atomic rows or planes [8], reduces the energy losses and scattering of ions [9] and results in a much longer penetration as compared with that in amorphous media at the same ion energy [10,11]. Lindhard [12] has shown that the lattice induced directional effects dominate at certain ranges of beam-to-target orientation when the incident angles $\psi_{\text {in }}$ with respect to axes or planes do not exceed the limitative critical angles $\psi_{\mathrm{c}}(E)$ known as the Lindhard angles of axial or planar channeling.

The ion implantation community places high emphasis on the channeling phenomenon [1]. Though primarily it was reckoned a valuable tool for deeper delivery of dopants associated with the reduced radiation damage of substrate $[6,13]$, currently [3] it is rated rather as a hindrance for the leading edge CMOS processes of the ultralarge scale integrated (ULSI) devices manufacturing. To obtain the ultra-shallow $p^{+} n$-junction, the sharpness of the transition from doped to undoped areas of a target is critical [14]. Due to channeling, the transition region is effectively diffused, so directional effects have to be suppressed as much as possible.

The pathway to avoid primary channeling is to tilt the wafer by angle $\psi_{\text {in }}>\psi_{\text {c }}$ off the axis (usually [001] for $\mathrm{Si}$ ) normal to the target surface. To prevent ions to be trapped into adjacent planar channels, wafers also are rotated by the angle $\phi_{\text {in }}$ with respect to the (011) plane. The twain $\left(\psi_{\text {in }}, \phi_{\text {in }}\right)$ defines the Random Equiva- 
lent Orientation (REO) of the off-axis implantation. The $\left(7^{\circ}, 30^{\circ}\right)$ REO is an established standard for mediumenergy boron implantation into $\mathrm{Si}$ in commercial implanters.

However, numerous off-axis irradiations [15-19] followed by the Secondary Ions Mass Spectrometry (SIMS) measurements of doping profiles clearly demonstrated the occurrence of directional effects. They still remain apparent as the observed long-range tails of $C(z)$ at $z \gg R_{\mathrm{p}}$. Using the MC simulation of ion penetration, Lever and Brannon [20] have explicitly identified these tails with the unintentional channeling in different (including high-index) axial channels.

At the depths of channeling tails, $C(z)$ tends to decrease exponentially i. e. much slower than the Gaussian decrease of $C(z)$ in disordered materials. Such a behavior constraints the sharpness of $p^{+} n$-junctions. To improve it, low-energy beams are generally favorable because of smaller $\delta R_{\mathrm{p}}(E)$. However, channeling in open axial channels survives even below $1 \mathrm{keV}$ [21], and the probability to penetrate behind the "amorphous" Gaussian profile definitely increases with the decrease of ion energy, and below $20 \mathrm{keV}$ reaches $\sim 20 \div 30 \%[16,17]$.

Generally, the channeling tails are currently recognized as an unavoidable feature of the low energy ion implantation into crystals, and physical mechanisms of their formation are of great interest. The conceptual progress in this field can be achieved within the framework of the analytical theory of directional effects $[7,12]$ that evaluates the stability of directed motion $[12,22]$ and the kinetics of channeling affected by various dechanneling factors of stochastic nature [7,23,24].

The goal of the present paper is to make a bridge between the complete 3D atomistic simulation and the channeling theory having in mind the development of the physically based model to determine the optimal REO conditions for off-axis implantation as functions of ion mass and energy. To that end, we perform the MD modeling of the off-axis doping profiles, correlate them with atomistic simulation and theory of directional effects in ion stopping, and obtain the explicit analytical criterion of the channeling tail minimization formulated as a new critical angle that extends the conventional Lindhard's treatment.

\section{SIMULATION METHODS AND MODELS}

Numerous 3D MC codes are currently applied to the simulation of ion implantation into crystals, including the computationally efficient binary collision approximation (BCA) codes (Crystal-TRIM [25], UTMARLOWE [26], MCIMPL-II [27], ALPHA TAURI [28]) and the high-precision molecular dynamics (MD) codes like MDRANGE [29] or REED-MD [30].

But none of these codes includes description of the physical parameters of particles within the scope of the channeling theory. Therefore, the original simulation tools of $3 \mathrm{D}$ ion transport have been developed to fit the problem of our interests.

\section{A. The MICKSER code}

The in-house developed computer code MICKSER $[24,31]$ performs the MC modeling of the 3D transport of fast ions of keV-to-MeV energies in layered structures of single crystals, polycrystalline and amorphous media as well as in certain kinds of nanostructures [32]. It is particularly oriented on the simulation of directional effects in ion implantation [24,32-35] and surface scattering [36].

The target crystallography is described by the user supplied lattice 3D basis vectors and unit cell configuration. Polycrystals are simulated using the procedure of the lattice random 3D rotations. Amorphous targets are represented as structureless random ensembles of atoms of chosen density. The layered target setup allows simulation of a large variety of interface effects such as, for example, effect of surface oxide films on doping profiles.

The essential feature of the MICKSER code is its capability to simulate 3D transport of ions using both the BCA and MD methods applicable to the same modeling task. The BCA algorithms of the code follow the basic guideline of other BCA codes [37] with special emphasis on the simulation of simultaneous collisions with atoms that is crucial for accurate channeling calculations. The BCA issues of collective interactions [37] are intrinsically eliminated by the MD simulation method that accounts for particle interactions with all atoms surrounded within the large enough interaction length $R_{\max }$. Trajectories are built by numerical solution of motion equations using the stable Verlet finite-difference scheme.

The code is focused on the fast particles dynamics simulation and thus implements the performance optimized Recoil Interaction Approximation (RIA) MD method [29]. It is a restricted metastable version of MD that takes into account only the atomic interactions of an energetic particle while the interactions between target atoms are ignored. For this reason the RIA MD method fails to model the lattice relaxation down to thermal energies and the formation of lattice defects. It is applicable to particle energies above $1 \div 10 \mathrm{eV}$. This restriction does not affect the kinetics of $\mathrm{keV}$ ions. The validity of the RIA method for ion implantation studies had been justified during extensive testing [29]. It offers efficiency perfectly comparable with that of BCA model and allows to simulate long-range phenomena (such as channeling) without the need to store huge crystallite data in computer memory.

The ion-atomic interaction is described by the general form of the repulsive central screened Coulomb potential:

$$
V(r)=\frac{Z_{1} Z_{2} e^{2}}{r} \Phi\left(\frac{r}{a}\right),
$$

where $Z_{1}$ and $Z_{2}$ are the atomic numbers of the incident ion and target atom separated by the distance $r$, $e$ is the electron charge. Different representations of the screening function $\Phi$ with appropriate screening length $a$ can be used, e. g. taking Lindhard, Moliere, or ZieglerBiersack-Littmark (ZBL) parametrizations [4, 12,37]. 
The thermal vibrations of atoms at temperature $T$ are taken into account introducing the uncorrelated random atomic displacements around the lattice sites. According to the Debye model, they are normally distributed with the mean-square amplitude $\left\langle u_{\mathrm{T}}^{2}\right\rangle=$ $\frac{3 \hbar^{2}}{M_{2} k_{\mathrm{B}} \Theta_{\mathrm{D}}}\left(\tau^{2} \int_{0}^{1 / \tau} \frac{x d x}{e^{x}-1}+\frac{1}{4}\right)$ where $M_{2}$ is the target atom mass, $\hbar$ and $k_{\mathrm{B}}$ are Planck and Boltzmann constants, $\Theta_{\mathrm{D}}$ is the Debye temperature, $\tau=T / \Theta_{\mathrm{D}}$. Obviously, the static $\left(\left\langle u_{\mathrm{T}}^{2}\right\rangle=0\right)$ lattices can be also simulated.

The inelastic electronic energy losses are represented in the code as a sum of the local and nonlocal parts [37]. The former one is dominant at close ion-atomic collisions and is described by the Firsov electronic stopping model [38] that leads to the following expression [39] for the interatomic friction force $\mathbf{F}_{i j}$ :

$$
\begin{aligned}
\mathbf{F}_{i j} & =\frac{2^{1 / 3} \hbar}{2 \pi a_{0}^{2}}\left(\mathbf{v}_{j}-\mathbf{v}_{i}\right)\left[Z_{A}^{2} I\left(\frac{Z_{A}^{1 / 3} \alpha r_{i j}}{0.8856 a_{0}}\right)\right. \\
& \left.+Z_{B}^{2} I\left(\frac{Z_{B}^{1 / 3}(1-\alpha) r_{i j}}{0.8856 a_{0}}\right)\right]
\end{aligned}
$$

where $a_{0}$ is the Bohr atomic radius, $\mathbf{v}_{i, j}$ are the velocities of $i^{\text {th }}$ and $j^{\text {th }}$ atoms at the distance $r_{i j}, Z_{A}=$ $\max \left(Z_{i}, Z_{j}\right), Z_{B}=\min \left(Z_{i}, Z_{j}\right), \alpha=\left[1+\left(Z_{B} / Z_{A}\right)^{1 / 6}\right]-1$, and $I(y)=\int_{y}^{\infty} \Phi^{2}(x) \frac{d x}{x}$. At RIA MD calculations, $\mathbf{F}_{i j}$ is methodically taken into account along with the conventional ion-atomic forces $\propto \nabla V_{i j}\left(r_{i j}\right)$.

The nonlocal part is described by the one-parameter model proposed by Cai et al. [40]. It is based on the Brandt-Kitagawa effective charge model [41]. The nonlocal energy loss $\Delta E_{\text {nonlocal }}$ is calculated by path integration over the ion trajectory $\mathbf{r}(t)$ :

$$
\begin{aligned}
\Delta E_{\text {nonlocal }} & =\left[Z_{1}^{\mathrm{eff}}\left(v, r_{\mathrm{s}}^{(0)}\right)\right]^{2} \\
& \times \int S_{\mathrm{p}}\left(v(\mathbf{r}), r_{\mathrm{s}}(\mathbf{r})\right) d \mathbf{r},
\end{aligned}
$$

where $Z_{1}^{\text {eff }}$ is the effective charge of an ion of the velocity $v, S_{\mathrm{p}}$ is the electronic stopping power of protons having the same velocity, $r_{\mathrm{s}}(\mathbf{r})=\left[3 / 4 \rho_{\mathrm{e}}(\mathbf{r})\right]^{1 / 3}$ is the oneelectron radius of the target atom, and $\rho_{\mathrm{e}}(\mathbf{r})$ is the $3 \mathrm{D}$ distribution of the target charge density. The last one can be obtained either by superposition of electron densities of isolated atoms (IADS approximation) or, more adequately, by ab initio calculations. The length $r_{\mathrm{s}}^{(0)}$ is the only free parameter of the model.

The code also takes into account the ion multiple scattering (MS) by target electrons. At each step $\delta z$ of the trajectory modeling, the MS induced angular deflection $\delta \theta$ of an ion is sampled from the Gaussian probability distribution function with zero mean value and the variance equal to the mean-square angle of MS [13]:

$$
\left\langle\delta \theta^{2}\right\rangle=\frac{\sqrt{5 m_{\mathrm{e}} v^{2} \hbar \omega_{\mathrm{p}}}}{4 E^{2}} \cdot \Delta E_{\text {nonlocal }}(\delta z)
$$

where $m_{\mathrm{e}}$ is the electron mass, $\hbar \omega_{\mathrm{p}}$ is the plasmon energy for the mean electron density of the target. The MS effects are usually weak as compared with nuclear scattering. However, they become considerable in the case of channeling when the nuclear scattering is strongly reduced.

\section{B. The channeling specific calculation methods}

To analyze the fast particles dynamics in crystals in terms of the channeling theory, the MICKSER code implements various on-line procedures. When they are activated in the modeling task, at each step of the ion trajectory simulation the code performs: (i) the search of the axial channels adjacent to the instantaneous direction of ion motion, (ii) calculations of transverse energies of axially channeled ions, and (iii) the evaluation of critical parameters taken from the channeling theory models.

The contiguous axial channels are searched by comparison of an ion velocity vector with crystallographic directions built from a target $3 \mathrm{D}$ lattice basis vectors. Typically a limited set of low-index neighboring channels is stored. The $2 \mathrm{D}$ orthogonal basis of atomic strings and the string sublattice coordinates are built immediately after the appropriate axial direction has been found. The thermally averaged continuum string potential $U[12,42]$ at a distance $r_{\perp}$ from the atomic row with spatial period $d$ is calculated using the ion-atomic potential $V(r)$ :

$$
\begin{aligned}
U\left(r_{\perp}\right) & =\frac{2}{d u_{\perp}^{2}} \int_{0}^{2 \pi} d \varphi \int_{0}^{\infty} e^{-\frac{r^{2}}{u_{\perp}^{2}}} r d r \\
& \times \int_{-\infty}^{+\infty} V\left(\sqrt{r_{\perp}^{2}+r^{2}-2 r_{\perp} r \cos \varphi+z^{2}}\right) d z .
\end{aligned}
$$

It is applied to calculate the continuum potential $U_{\text {ch }}$ of the axial channel at transversal coordinates $\mathbf{r}_{\perp}(x, y)$ by superposition of continuum potentials of atomic rows arranged in the $2 \mathrm{D}$ lattice $\mathbf{r}_{\perp}^{(i)},(i=1,2, \ldots)$ of the transversal plane:

$$
U_{\mathrm{ch}}\left(\mathbf{r}_{\perp}\right)=\sum_{\left|\mathbf{r}_{\perp}-\mathbf{r}_{\perp}^{(i)}\right|<R_{\max }} U\left(\left|\mathbf{r}_{\perp}-\mathbf{r}_{\perp}^{(i)}\right|\right)
$$

Here $R_{\max }$ is the cut-off interaction length, and $u_{\perp}^{2}=$ $\frac{2}{3}\left\langle u_{\mathrm{T}}^{2}\right\rangle$.

A fundamental quantity of the channeling theory, the transverse energy $E_{\perp}$ of an ion having a small angle $\psi$ to the channel axis at the point $\mathbf{r}_{\perp}$ of the transversal plane is calculated as a sum of the kinetic $\left(E \sin ^{2} \psi \approx E \psi^{2}\right)$ and potential terms:

$$
E_{\perp}=E \sin ^{2} \psi+U_{\operatorname{ch}}\left(\mathbf{r}_{\perp}\right) .
$$

It is conserved at transversal motion in the $2 \mathrm{D}$ potential (6). To avoid non-physical oscillations, at 3D MD modeling $E_{\perp}$ is running-averaged over the spatial period $d$, the minimal longitudinal extent of the continuum potential $U_{\mathrm{ch}}\left(\mathbf{r}_{\perp}\right)$ applicability. 
For ions, the classically accessible area is confined by the condition $U_{\mathrm{ch}}\left(\mathbf{r}_{\perp}\right) \leq E_{\perp}$. The equipotential line $\mathbf{r}_{\perp \text { min }}$ resolving the equation $U_{\mathrm{ch}}\left(\mathbf{r}_{\perp \text { min }}\right)=E_{\perp}$ contains the points of the closest $(\psi=0)$ approach of an ion to atomic rows. In the vicinity of an atomic row the closest approach distance $r_{\perp \text { min }}$ can be estimated in a single-string approximation (SSA) by the equation $U\left(r_{\perp \text { min }}\right)=E_{\perp}$ since $U_{\mathrm{ch}}\left(\mathbf{r}_{\perp}\right) \approx U\left(r_{\perp}\right)$ at small $r_{\perp}$.

Critical parameters [12] restrict transverse energies, incident angles and energies of ions that fulfill conditions of the continuum potential applicability. The critical transverse energy $E_{\perp \mathrm{c}}$ and angle $\psi_{\mathrm{c}}=\sqrt{E_{\perp \mathrm{c}} / E}$ of stable axial channeling are defined by the value of $U_{\mathrm{ch}}$ at the critical point $\mathbf{r}_{\perp_{c}}$ where channeling stability is broken:

$$
E_{\perp \mathrm{c}}(E)=E \psi_{\mathrm{c}}^{2}(E)=U_{\mathrm{ch}}\left(\mathbf{r}_{\perp \mathrm{c}}(E)\right) .
$$

The point $\mathbf{r}_{\perp_{\mathrm{c}}}$ corresponds to the critical distance $r_{\perp \mathrm{c}}$ of the closest approach of an ion to atomic rows. Several modes of transversal motion co-exist in 2D lattices of atomic strings. The code assumes that the critical parameters are determined by the motion in a plane containing the point $\mathbf{r}_{\perp}^{*}$ of local minimum of $U_{\mathrm{ch}}$ and the nearest to $\mathbf{r}_{\perp}^{*}$ atomic row. With this assumption, the smallest value of $E_{\perp \mathrm{c}}$ is obtained.

The channeling stability criteria solved for the critical distances $r_{\perp c}$ of the closest approach of an ion to atomic rows are the basic entities of this method of the critical parameters evaluation. The MICKSER code implements different criteria. The first of them is the generalized [22] Lindhard condition [12] of the loss of correlation between successive ion-atomic collisions at glancing scattering by atomic row. It follows from the non-conservation of $E_{\perp}$ at $r_{\perp}<r_{\perp \mathrm{c}}^{(\mathrm{L})}$ and has the form:

$$
d^{2} \cdot U_{\mathrm{ch}}^{\prime \prime}\left(r_{\perp \mathrm{c}}^{(\mathrm{L})}(E)\right)=8 E
$$

where the primes denote the derivation along the direction from the nearest axis to the point $\mathbf{r}_{\perp}^{*}$ that allows to account for actual structure of axial channels.

The second criterion describes the appearance, at $r_{\perp}<$ $r_{\perp \mathrm{c}}^{(\mathrm{S})}$, of the parametric instability [43] of the particle motion in the channels of finite transversal size. The correspondent criterion equation is the following [22]:

$$
d^{2} \cdot\left[U_{\mathrm{ch}}^{\prime}\left(r_{\perp \mathrm{c}}^{(\mathrm{S})}(E)\right)\right]^{2}=4 E \cdot U_{\mathrm{ch}}\left(r_{\perp \mathrm{c}}^{(\mathrm{S})}(E)\right) .
$$

It is more restrictive than Eq. (9) and yields larger $r_{\perp \mathrm{c}}^{(\mathrm{S})}>r_{\perp \mathrm{c}}^{(\mathrm{L})}$ and smaller $E_{\perp \mathrm{c}}$.

In $\operatorname{SSA}^{-\mathrm{c}}\left(U_{\mathrm{ch}} \simeq U\right)$ valid at sufficiently small $r_{\perp \mathrm{c}}$ Eq. (9) reduces to the conventional Lindhard criterion [12, Appendix I] consistent with the well-known Lindhard critical angles. For the characteristic value $U_{\mathrm{s}}=2 Z_{1} Z_{2} e^{2} / d$ of the axial potential barrier and the standard static continuum potential

$$
U_{\mathrm{L}}\left(r_{\perp}\right)=U_{\mathrm{s}} \cdot \ln \sqrt{1+\frac{3 a^{2}}{r_{\perp}^{2}}}
$$

Lindhard [12] has estimated that $\psi_{\mathrm{c}}(E) \simeq \psi_{1}(E)=$ $\sqrt{\frac{U_{\mathrm{s}}}{E}} \propto E^{-\frac{1}{2}}$ at high energies $E>E^{\prime}=\left(\frac{d}{a}\right)^{2} U_{\mathrm{s}}$ while $\psi_{\mathrm{c}}(E) \simeq \psi_{2}(E)=\left(\frac{3 a^{2} U_{\mathrm{s}}}{2 d^{2} E}\right)^{\frac{1}{4}} \propto E^{-\frac{1}{4}}$ at low $E<E^{\prime}$.

These considerations and Eq. (8) show that $E_{\perp \mathrm{c}} \simeq U_{\mathrm{s}}$ is energy independent at high $E>E^{\prime}$ where $r_{\perp c}<a \sim$ $u_{\perp}$ since the continuum approximation definitely fails due to thermal vibrations of atoms. On the other hand, $E_{\perp \mathrm{c}}=E \psi_{2}^{2} \propto E^{-\frac{1}{2}}$ at energies of ion implantation, $E<E^{\prime}$, where the value of $r_{\perp \mathrm{c}}(E)>a$ and increases as $E$ decreases. At threshold energy $E_{\text {ch }} \ll E^{\prime}$ it reaches the characteristic radius $r_{0}=(\pi n d)^{-\frac{1}{2}}$ of a channel (here $n$ is the target atomic density). It means that no channeling is expected to occur below $E_{\mathrm{ch}}: E_{\perp \mathrm{c}}(E) \equiv 0$ and $\psi_{\mathrm{c}}(E) \equiv 0$ at $E \leq E_{\mathrm{ch}}$.

The code estimates the energy thresholds for each channeling stability criterion by the calculation of the ion energy at which $\mathbf{r}_{\perp_{\mathrm{c}}} \rightarrow \mathbf{r}_{\perp}^{*}$. The correlation loss criterion (9) yields $E_{\mathrm{ch}}=E_{\mathrm{ch}}^{(\mathrm{L})}=\frac{1}{8} d^{2} \cdot U_{\mathrm{ch}}^{\prime \prime}\left(\mathbf{r}_{\perp}^{*}\right)$. For Eq. (10), $E_{\mathrm{ch}}=E_{\mathrm{ch}}^{(\mathrm{S})}=4 E_{\mathrm{ch}}^{(\mathrm{L})}[22]$, thus the parametric instability of channeling is always dominant ${ }^{1}$.

The generic methods of critical parameters calculations implemented in the MICKSER code can be applied to evaluate the critical conditions not only for channeling itself but for whatever impact parameter dependent processes if the correspondent criterion equations are properly identified.

Finally one should note that if several open $\left(E_{\text {ch }}<\right.$ $E$ ) axial channels had been found at the stage (i) of MICKSER algorithms then the channel having the minimal $E_{\perp} / E_{\perp c}$ ratio is selected as a candidate one the ion to be channeled in. The ion is considered as stably channeled if $E_{\perp}<E_{\perp c}$. It allows to discriminate directed and chaotic modes of an ion's motion, to calculate the channeling probabilities at various depths (the dechanneling function $P_{\mathrm{ch}}(z)$ ), the distributions in $E_{\perp}$ and other quantities and functions that describe the kinetics of ion transport in crystals.

\section{THE PROBLEM FORMULATION}

The ranges $R_{\mathrm{p}}\left(E_{\mathrm{in}}\right) \approx \int_{0}^{E_{\mathrm{in}}} \frac{d E}{|d E / d x|}$ of ions of energy $E_{\text {in }}$ are influenced in crystals by the lattice driven variations of specific energy losses $|d E / d x|$. At dynamically stable channeling, the flux-peaking effect $[12,44]$ reduces $|d E / d x|$ of ions $[6,42]$ as compared with $|d E / d x|_{\mathrm{a}}$, the stopping in a structureless amorphous solid [4]. It is usually adopted that $|d E / d x| \simeq|d E / d x|_{\text {a }}$ at non-channeled

\footnotetext{
${ }^{1}$ However, for off-axis low-energy ion implantation the unstable channeling is rather significant. Hence, hereinafter the critical parameters determined by Eq. (9) are systematically used.
} 
incidence $\left(\psi_{\text {in }}>\psi_{\mathrm{c}}\right)$. Therefore, the Lindhard angle $\psi_{\mathrm{c}}$ is considered as a barrier of directional dependence of ion stopping, and the doping profile in a crystalline target is completely determined by the kinetics of transitions between channeled and random modes.

However, more refined angular dependencies of $|d E / d x|$ in the above-barrier region $\psi_{\text {in }}>\psi_{\mathrm{c}}$ have been revealed in numerous experiments with highly collimated ion beams [6], including azimuthal dependencies on $\phi_{\text {in }}$ (that are due to non-equilibrium effects of particles captured into planar channels) and the presence of a component with abnormally high energy losses [42]. For implantation, Myers et al. [15] have found experimentally that the best REO tilt angles for a range of ion species and energies are about twice as large as the critical angles $\psi_{\mathrm{c}}$ estimated according to Lindhard's theory. They hit upon an angular range referred in theory as the "quasichanneling" $[45,46]$ and associated with the enhanced nuclear scattering and energy losses. This suggests that a significant role in the formation of channeling tails is played by the dynamically unstable above-barrier states of ion motion.

The above-barrier motion is mostly considered at relativistic energies [47] when the continuum potentials of atomic rows are applicable up to $\psi \sim(a / d)$ that is much greater than the relativistic Lindhard angle $\psi_{\mathrm{L}}=\sqrt{2 U_{\mathrm{s}} / E}$. In turn, quasichanneling $[45,46]$ is usually attributed to $\sim \mathrm{MeV}$ energy light ions mainly concerning the particles focusing at close collisions with atoms. At $E>E^{\prime}$ it is closely related to the blocking effect [48] and has the same characteristic angle, $\psi_{\mathrm{b}}>\psi_{\mathrm{c}}$, that determines the opening radius of the shadow cone for a recoil emerging from the lattice site along the close-packed lattice direction.

But at implantation $(\sim \mathrm{keV})$ energies no extensive studies of quasichanneling or of above-barrier motion of heavy ions was made due to experimental difficulties. The theoretical consideration is also hindered since $\psi_{\mathrm{b}}>\psi_{\mathrm{c}}[12,48]$ and the continuum approximation cannot describe quasichanneling. Consequently, the 3D computer simulation technique is the most appropriate method for these studies.

We shall apply this technique, implemented in the MICKSER RIA MD code, to clarify the role of unstable above-barrier modes of ion motion in the formation of channeling tails at off-axis implantation. We consider the implantation of boron $\left({ }_{5} \mathrm{~B}\right)$ and arsenic $\left({ }_{33} \mathrm{As}\right)$ ions of the same initial energy, $15 \mathrm{keV}$, into the silicon $\left({ }_{14} \mathrm{Si}\right)$ single crystal at the standard REO $\left(7^{\circ}, 30^{\circ}\right)$ incidence onto a (001) surface plane. Both implants are typical for industrial technologies but strongly differ in the projectileto-target atomic mass ratio $M_{1} / M_{2}(0.385$ and 2.667, respectively).

At $15 \mathrm{keV}$, the Lindhard critical angles $\psi_{\mathrm{c}} \simeq \psi_{2}$ calculated for the major $\langle 001\rangle$ atomic row are equal to $4.3^{\circ}$ and $5.9^{\circ}$, respectively for $\mathrm{B}$ and As. Thus at the tilt angle $\psi_{\text {in }}=7^{\circ}>\psi_{\text {c }}$ no surface capture in the $\langle 001\rangle$ stable channeling is expected for both implants. In order to distinguish the volume capture effects from those caused by the ion beam angular spread and the scattering by target surface contamination, the mono-directional non-divergent beam and the absence of the surface oxide amorphous layer were knowingly assumed in our simulation.

The "universal" ZBL [4] interaction potential is used for both $\mathrm{B}$ and As ions having the screening length $a$ of $0.0143 \mathrm{~nm}$ and $0.0115 \mathrm{~nm}$, respectively. The cut-off interaction length $R_{\max }=0.2715 \mathrm{~nm}$ was chosen for $\mathrm{Si}$ diamond type lattice.

Since thermal displacements of lattice atoms affect essentially the kinetic of low-energy ions axial channeling $[7,23,24]$ the modeling was carried out both for regular static $\left(\left\langle u_{\mathrm{T}}^{2}\right\rangle=0\right)$ lattice and for thermally vibrating lattice at $T=300 \mathrm{~K}$. The r.m.s. amplitude $\sqrt{\left\langle u_{\mathrm{T}}^{2}\right\rangle}=$ $0.0079 \mathrm{~nm}$ has been derived from the up-to-date data on Si single crystal Debye temperature $\Theta_{\mathrm{D}}=519 \mathrm{~K}$ [49].

For the parameters of the nonlocal electron stopping model (3) of both implants we adopted the recommended [40] value of $r_{\mathrm{s}}^{(0)}=1.9 a_{0}=0.10054 \mathrm{~nm}$. The $3 \mathrm{D}$ distribution of electron density $\rho_{\mathrm{e}}(\mathbf{r})$ of Si crystal has been calculated by means of the $a b$ initio code CRYSTAL2003 [50].

The model parameters of the MICKSER code MD simulation had been validated earlier $[24,33]$ and had been found to reproduce fairly the experimental data on the as-implanted doping profiles at the channeling incident of B and As ions into Si, as well as the SRIM2006 code calculated profiles in amorphous silicon (a-Si).

\section{SIMULATION OF CHANNELING TAILS}

The REO doping profiles $C(z)$ calculated by the MICKSER code are compared in Fig. 1 with the SIMS measured profiles [40] and the SRIM2006 code calculated profiles of implantation into a-Si at $7^{\circ}$ tilt with respect to the surface normal.

At $15 \mathrm{keV}$, the SRIM2006 code reports the range and straggling $\left(R_{\mathrm{p}} \pm \delta R_{\mathrm{p}}\right)$ in a-Si to be equal to $(58.7 \pm 31) \mathrm{nm}$ for $\mathrm{B}$ and $(16.1 \pm 5.8) \mathrm{nm}$ for As ions. In Fig. 1, the comparison with SRIM data testifies the evidence of longrange exponential tails of $C(z)$ at depths $z \gg R_{\mathrm{p}}$ for both kinds of ions and both in static and thermally vibrating lattices. As compared to $R_{\mathrm{p}}$ in a-Si, heavier ions (As) reach about twice as large depth $z \simeq 8 R_{\mathrm{p}}$ than boron having tails only up to $z \simeq 4 R_{\mathrm{p}}$.

Qualitatively, the simulation results agree well with the available experimental data. The observed quantitative deviation at large $z$ is due to the implanter beam angular divergence $\left(\sim 0.5^{\circ}\right)$ and the presence of $\mathrm{a} \sim 1.5 \mathrm{~nm}$ thick amorphous $\mathrm{SiO}_{2}$ surface layer [40]. Both these experimental factors favor the scattering of ions into the major [001] axial channel near the crystal surface. Their incorporation into the MICKSER simulation model completely matches the calculated and SIMS profiles. However, in Fig. 1 we have presented the simulation results for the idealized (zero divergence, perfect crystal) setup to demonstrate that the appearance of long-range tails is caused by the ordered crystalline structure of the target. 


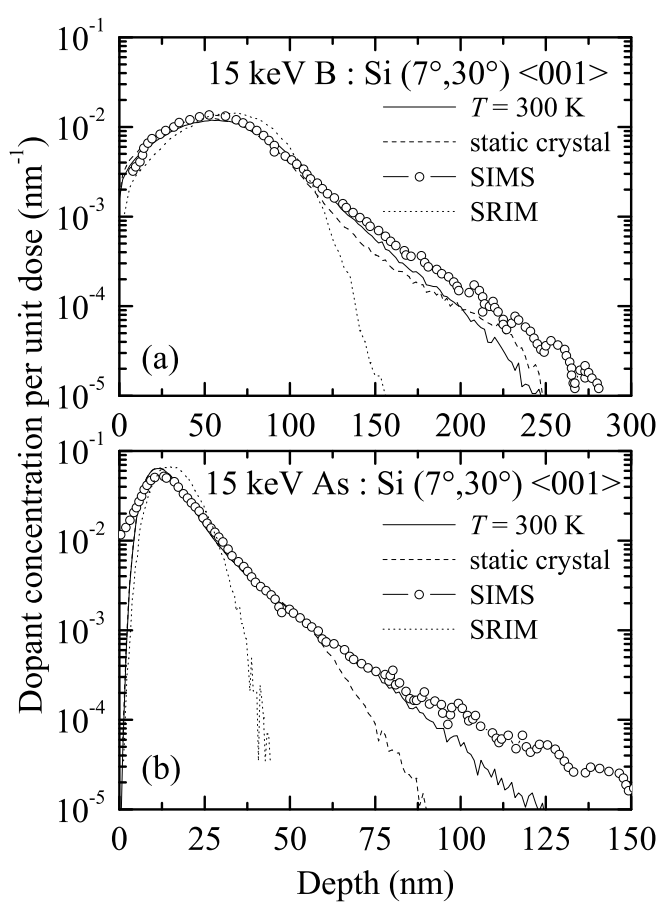

Fig. 1. The simulated as-implanted profiles of $15 \mathrm{keV}$ B (a) and As (b) ions off-axis REO $\left(7^{\circ}, 30^{\circ}\right)$ implantation into perfect (001)Si crystals at different amplitudes of atomic thermal vibrations in comparison with standard implantation profiles into $7^{\circ}$ tilted amorphous Si targets (SRIM [5]) and SIMS experimental data [40].

The distributions of ions in the reduced transverse energy $E_{\perp} / E_{\perp \mathrm{c}}$ with respect to the major [001] axial channel are shown in Fig. 2. To obtain them, for each ion that did not stopped before the depth $z$, the ratio $E_{\perp}(E, \psi) / E_{\perp \mathrm{c}}(E)$ was calculated by the MICKSER code using, as described in Sec. II B, Eqs. (7-9) and the current values of the ion's energy $E(z)$ and angle $\psi(z)$. The application of the conventional MC tally procedure resulted in the $\left(E_{\perp} / E_{\perp c}\right)$ distribution function. For each depth, it has been normalized to the total number of non-stopped ions and automatically accounts for their angular distribution and energy spectrum. Thus, the ions having $E_{\perp} / E_{\perp \mathrm{c}} \leq 1$ can be attributed to the stable channeling at depth $z$ taking into account the energy dependency of the Lindhard's critical parameters of channeling.

The near-surface distributions $\left(z \ll R_{\mathrm{p}}\right)$ are peaked at $E_{\perp} \approx 2.3 E_{\perp \mathrm{c}}$ for $\mathrm{B}$, and $E_{\perp} \approx 1.6 E_{\perp \mathrm{c}}$ for As. It justifies the lack of surface capture at the off-axis incidence.

For static crystal, the distributions of Fig. 2(a,b) demonstrate a fast decay at $E_{\perp}<E_{\perp c}$. The region $E_{\perp} \ll E_{\perp c}$ (commonly referred as hyperchanneling [6]) is practically vacuous in this case. It is due to the fact that in the absence of thermal vibrations the major factor that determines the channeled particle diffusion over $E_{\perp}$ is the discreteness of atomic rows. But as $E_{\perp}$ decreases, the effect of discreteness decreases exponentially [51], and becomes negligible at hyperchanneling. At the same time, the capture of ions into hyperchanneling as a result of single strong atomic scattering is totally prohibited by the blocking effect. The blocking of hyperchanneling is much more expressed for As ions that is most likely determined by a weaker sensitivity of the heavier ion to the discontinuity of atomic rows.
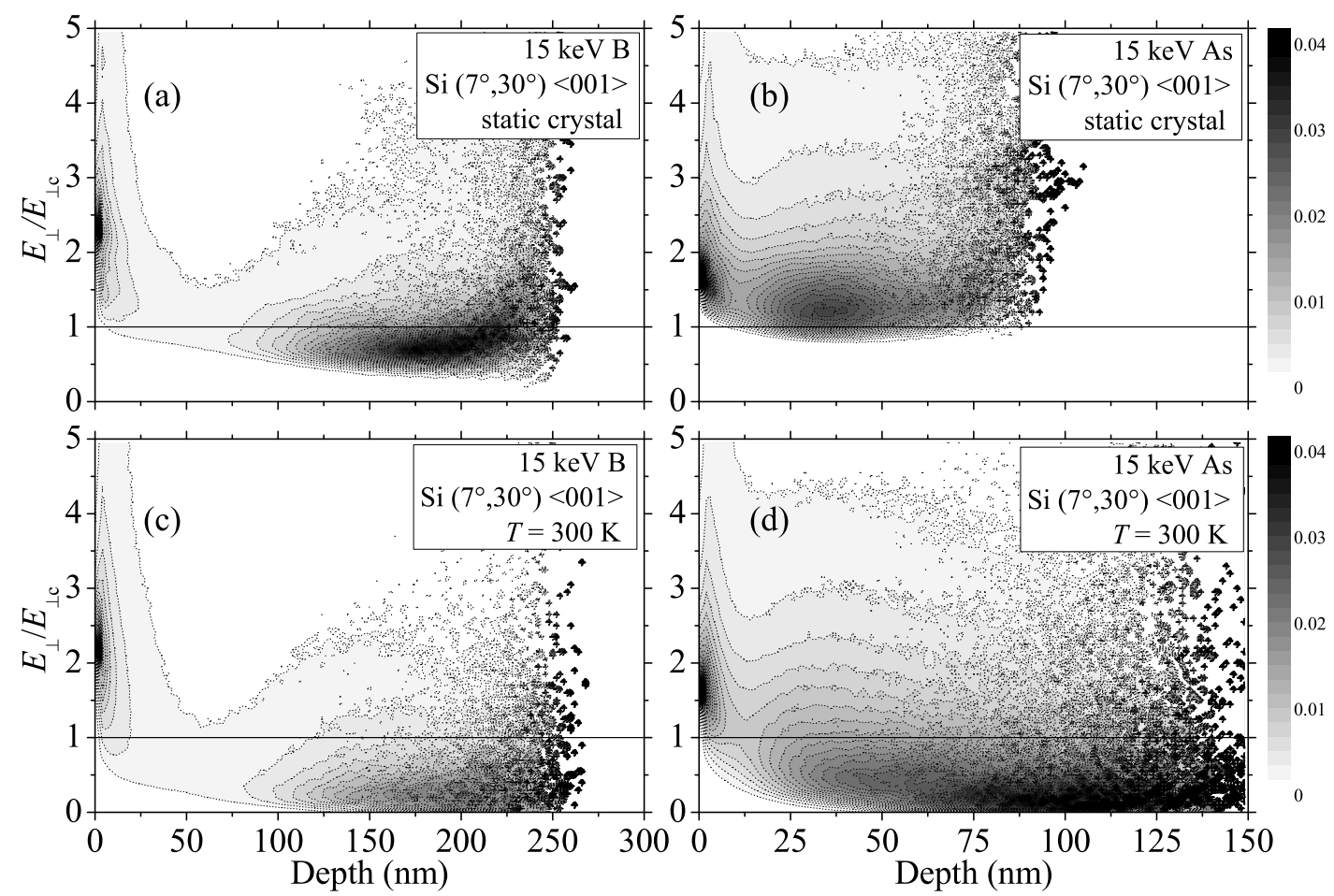

Fig. 2. Normalized distributions of non-stopped ions in the reduced transverse energy $E_{\perp} / E_{\perp c}$ as functions of penetration depth for static Si lattice $(\mathrm{a}, \mathrm{b})$ and at $T=300 \mathrm{~K}(\mathrm{c}, \mathrm{d})$. Off-axis implantation of $15 \mathrm{keV} \mathrm{B} \mathrm{(a,c)} \mathrm{and} \mathrm{As} \mathrm{(b,d)} \mathrm{ions.}$ 
At $T=300 K$, ions fill all ranges of $E_{\perp}$, including that of hyperchanneling. It means that thermal vibrations facilitate the volume capture of ions into the stable channeling mode. At large $z \gg R_{\mathrm{p}}$ the maximum of distribution shifts to lower $E_{\perp} / E_{\perp c}$, thus the relative population of the channeling states increases.

For heavier As ions, due to the flux-peaking induced reduction of nuclear energy losses the capture into the channeling mode leads to the substantial increase of the maximal range, and drastically affects the total length of the channeling tail as compared with that in a static crystal (see also Fig. 1(b)). For boron, the flux-peaking suppression of the prevailed electronic stopping is not so pronounced because of its weaker dependence on $E_{\perp}$. It is effectively compensated by the competitive increase of the dechanneling rate due to thermal vibrations. As a result, the temperature effect on the length of channeling tails is much weaker for lighter B ions.

The maps of Fig. 2 clearly demonstrate that, in contrast to the basic assumptions of Lindhard's theory, the critical transverse energy $E_{\perp c}$ of channeling actually is not a well-defined barrier that separates the modes of directed and random motion, especially in the case of a static crystal, or at low temperatures. For low-energy heavy ions implantation, the directional effects are traced up to $E_{\perp} \simeq(2 \div 3) \times E_{\perp c}$ attributed to the dynamically unstable transversal motion of ions.

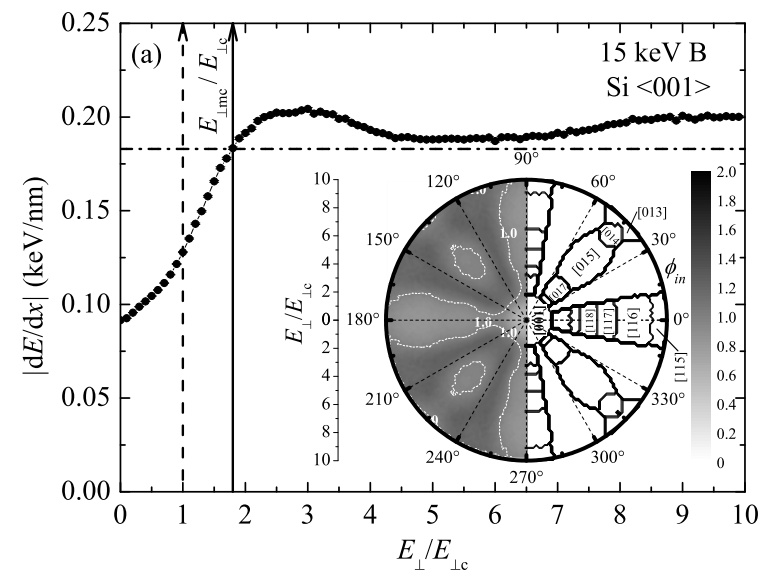

\section{DIRECTIONAL DEPENDENCIES OF ION STOPPING}

To reveal the origin of directional effects in doping profiles, one has to connect them with those of the underlying physical quantity, the energy losses of particles.

The directional dependencies of the specific energy losses $|d E / d x|$ in the neighborhood of the [001]Si channel are shown in Fig. 3 as functions of $E_{\perp}$ in units of the correspondent Lindhard critical transverse energy $E_{\perp c}$ of [001] channeling. To calculate them, the passage of $15 \mathrm{keV}$ ions through a thin $(\Delta z=3 d=1.629 \mathrm{~nm})$ crystal layer was modeled for a wide range of initial transverse energies $E_{\perp \text { in }}$ and uniformly distributed azimuthal incident angles $\phi_{\text {in }}$. The MC rejection method was used to provide the required value of $E_{\perp \text { in }}$ at each point $\mathbf{r}_{\perp \text { in }}$ of the particle incidence onto a transversal plane by adjusting $\psi_{\text {in }}$ according to Eq. (7). The transmission energy spectra have been analyzed, and the MC estimate $|d E / d x| \simeq\langle\Delta E / \Delta z\rangle$ was attributed to $E_{\perp \text { in }} / E_{\perp \mathrm{c}}$ and $\phi_{\text {in }}$. The curves of Fig. 3 represent the dependencies averaged over all possible $\phi_{\text {in }}$ while the details of dependencies on $\phi_{\text {in }}$ are shown in the inset plots. The horizontal dash-dotted lines indicate the rates of $|d E / d x|_{\mathrm{a}}$ of $15 \mathrm{keV}$ ions in a-Si $(0.183 \mathrm{keV} / \mathrm{nm}$ for $\mathrm{B}$ and $1.113 \mathrm{keV} / \mathrm{nm}$ for As) calculated according to the stopping database of the SRIM2006 $\operatorname{code}^{2}$.

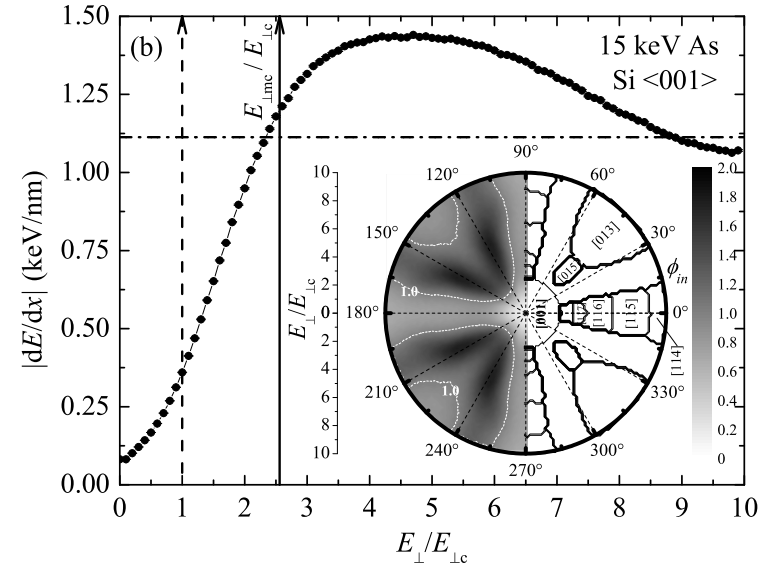

Fig. 3. The dependencies of the averaged over the azimuthal angle $\phi_{\text {in }}$ specific energy losses $|d E / d x|$ of $15 \mathrm{keV} \mathrm{B}$ (a) and As (b) ions on the reduced transverse energy $\left(E_{\perp} / E_{\perp c}\right)$ with respect to the [001]Si axial channel. The left parts of the inset plots demonstrate the raw data on the azimuthal dependencies of the ratio $\left(\left|\frac{d E}{d x}\right| /\left|\frac{d E}{d x}\right|_{\mathrm{a}}\right)$, the right parts are discussed in Sec. VI.

As it is seen in Fig. 3, at channeling $\left(E_{\perp} / E_{\perp \mathrm{c}}<1\right)$ the stopping rate of both ions is strongly suppressed. At hyperchanneling, the reduction factor is $\approx 2$ for $\mathrm{B}$, and about one order of magnitude for As. The prevalence of nuclear stopping of a heavier ion results in the smaller normalized minimum yield $\chi(0)=\left|\frac{d E}{d x}\right| /\left|\frac{d E}{d x}\right|_{\mathrm{a}}$.
On the contrary, the "shoulders" of enhanced $|d E / d x|$ are observed at large $E_{\perp} / E_{\perp \mathrm{c}}$. They are forming due to quasichanneling $[45,46]$. This effect is more expressed for heavier ions $\left(\chi_{\max } \simeq 1.3\right.$ for As and $\simeq 1.1$ for $\left.\mathrm{B}\right)$. The positions of the broad maxima of $|d E / d x|$ are in reasonable agreement with the transverse energies $E_{\perp} \simeq E \psi_{\mathrm{b}}^{2}$ de-

${ }^{2}$ MICKSER MD calculations result in the same values if the layer is built as structureless. 


\section{I. BRATCHENKO, A. S. BAKAI, S. V. DYULDYA}

rived from the [001] atomic row blocking angle $\psi_{\mathrm{b}}$ (at $15 \mathrm{keV}$, the calculations of $\psi_{\mathrm{b}}$ for the ZBL potential in use by means of the methods of refs. $[12,48]$ result in $E \psi_{\mathrm{b}}^{2} \simeq 2.35 E_{\perp \mathrm{c}}$ for $\mathrm{B}$ and $\simeq 3.92 E_{\perp \mathrm{c}}$ for As ions). However, the angular maps of Fig. 3 inset plots testify that at large $E_{\perp} / E_{\perp \mathrm{c}}>3$ the influence of the neighboring axial and planar channels cannot be ignored since $|d E / d x|$ becomes substantially anisotropic with respect to $\phi_{\text {in }}$. It is responsible for the observed deviations of $|d E / d x|$ from $|d E / d x|_{\mathrm{a}}$ at large $E_{\perp}$ where they have no asymptotical tendency to be in exact agreement (as it is usually assumed in the qualitative theory).

Concerning the effect of the stopping reduction on the formation of channeling tails, the key feature we find in Fig. 3 is the existence of a broad range of above-barrier $\left(E_{\perp}>E_{\perp \mathrm{c}}\right)$ transverse energies where the stopping rate still remains smaller than $|d E / d x|_{\mathrm{a}}$. It correlates to the extended width of the transverse energy distributions of implanted ions (see Fig. 2).

This range of dynamically unstable channeling occupies an intermediate position between the stable channeling and the quasichanneling. For short, we introduce the term "metachanneling" to identify this type of metastable directed motion of ions having $E_{\perp}>E_{\perp c}$. Its upper bound, $E_{\perp \mathrm{mc}}$, can be rated as a new critical parameter of directional effects, that is considered in more detail in the next section.

\section{THE CRITICAL PARAMETERS OF METACHANNELING}

According to Lindhard theory, directional effects at stable channeling $\left(E_{\perp}<E_{\perp c}\right)$ are caused by the fact that only far ion-atomic collisions with impact parameters $p>r_{\perp \mathrm{c}}$ contribute to particle stopping and induced secondary effects. Close $\left(p<r_{\perp c}\right)$ collisions are forbidden dynamically at stable channeling since far collisions conserve the transverse energy. It is consistent with the applicability of the continuum potential approximation.

At $E_{\perp}>E_{\perp \mathrm{c}}$, close collisions are no longer forbidden, and the continuum approximation fails. However, at metachanneling it still remains applicable at those sections of trajectory where a particle moves at the distances $r_{\perp}>r_{\perp \mathrm{c}}$ from the nearest atomic row and experience only far collisions with reduced stopping.

We show below that the mean length $\lambda_{\text {mc }}$ of such a section decreases as $E_{\perp}$ increases. Thus, it is directionally dependent. It means that, despite its finite lifetime (metastability), this type of motion is nevertheless affecting the directional phenomena, as it has been observed in computer simulation of Sec. V.

Let's estimate the upper bound $E_{\perp \mathrm{mc}}>E_{\perp \mathrm{c}}$ of the channeling dip of $|d E / d x|$ where the specific energy losses $|d E / d x|_{\mathrm{mc}}$ of metachanneled ions are equal to $|d E / d x|_{\mathrm{a}}$, the stopping rate in an amorphous solid of the same atomic density $n$. At $E_{\perp}>E_{\perp \mathrm{c}}$ the ion stopping rate can be partitioned into the sum of contributions of far $\left(p>r_{\perp \mathrm{c}}\right)$ collisions with atoms and of strong collisions with atomic rows:

$$
\left|\frac{d E}{d x}\right|_{\mathrm{mc}}=\left|\frac{d E}{d x}\right|_{\mathrm{mc}}^{\left(p>r_{\perp \mathrm{c}}\right)}+\frac{\delta E_{\mathrm{close}}^{(\mathrm{mc})}}{\lambda_{\mathrm{mc}}}
$$

where $\delta E_{\text {close }}^{(\mathrm{mc})}$ is the mean energy loss at close $\left(p<r_{\perp \mathrm{c}}\right)$ collision with the atomic row. Similar partitioning can be formally made for $|d E / d x|_{\mathrm{a}}$ :

$$
\left|\frac{d E}{d x}\right|_{\mathrm{a}}=\left|\frac{d E}{d x}\right|_{\mathrm{a}}^{\left(p>r_{\perp \mathrm{c}}\right)}+\frac{\delta E_{\text {close }}^{(\mathrm{a})}}{\lambda_{\mathrm{a}}}
$$

where $\delta E_{\text {close }}^{(\mathrm{a})}$ and $\lambda_{\mathrm{a}}^{-1}$ are the mean energy loss and the macroscopic cross-section of a close atomic collision having the microscopic cross-section $\sigma_{\mathrm{c}}=\pi r_{\perp \mathrm{c}}^{2}$ :

$$
\lambda_{\mathrm{a}}^{-1}(E)=n \sigma_{\mathrm{c}}(E)=n \pi r_{\perp \mathrm{c}}^{2}(E) .
$$

In our model, two basic assumptions have been made to compare Eq. (12) with Eq. (13). Both had been validated at MD simulation of the ion scattering by atomic rows [34]. First, the impact parameters distribution of far glancing collisions of metachanneled ions does not differ from that of random far collisions in amorphous medium. Therefore, $|d E / d x|_{\mathrm{mc}}^{\left(p>r_{\perp \mathrm{c}}\right)}=|d E / d x|_{\mathrm{a}}^{\left(p>r_{\perp \mathrm{c}}\right)}$. Next, at a close critical $\left(r_{\perp}>r_{\perp \mathrm{c}}\right)$ collision of a heavy above-barrier ion with atomic row the energy loss is determined by a single atomic scattering. Consequently, $\delta E_{\text {close }}^{(\mathrm{mc})}=\delta E_{\text {close }}^{(\mathrm{a})}$.

Therefore, the difference between $|d E / d x|_{\mathrm{a}}$ and $|d E / d x|_{\text {mc }}$ is due to the difference in macroscopic crosssections $\lambda_{\mathrm{a}}^{-1}$ and $\lambda_{\mathrm{mc}}^{-1}$. The former one is attributed to $3 \mathrm{D}$ random atomic collisions while the latter one corresponds to $2 \mathrm{D}$ close collisions of the metachanneled ions with atomic rows of an ordered lattice. Assuming these collisions to be uncorrelated (as in the dynamical chaos approximation [47]), it can be written in the form $\lambda_{\mathrm{mc}}^{-1}=n_{\perp} \sigma_{\perp} \psi$ where $n_{\perp}=n d$ is the spatial density of atomic rows, $\psi=\sqrt{E_{\perp} / E}$, and $\sigma_{\perp}$ is the microscopic cross-section of an ion $2 \mathrm{D}$ scattering by an atomic row having the distance of the closest approach $r_{\perp \text { min }} \leq r_{\perp \mathrm{c}}$. For transversal scattering, the cross-section $\sigma_{\perp}$ has the dimension of length, and equals to $2 b_{\mathrm{c}}$ where $b_{\mathrm{c}}$ is the atomic string impact parameter that matches $r_{\perp \text { min }}$ with $r_{\perp \mathrm{c}}$. Since at $r_{\perp} \geq r_{\perp \mathrm{c}}$ the continuum potential $U$ is still applicable, $b_{\mathrm{c}}$ obeys the equation of radial transversal motion governed by $U\left(r_{\perp}\right)$. Therefore, $1-\frac{U\left(r_{\perp \mathrm{c}}\right)}{E_{\perp}}-\left(\frac{b_{\mathrm{c}}}{r_{\perp \mathrm{c}}}\right)^{2}=0$ at the turning point $r_{\perp \min }=r_{\perp \mathrm{c}}$. Taking into account that $U\left(r_{\perp \mathrm{c}}\right)=E_{\perp \mathrm{c}}$, one can easily obtain from this equation that $\sigma_{\perp}=2 r_{\perp \mathrm{c}} \sqrt{1-E_{\perp \mathrm{c}} / E_{\perp}}$, and

$$
\lambda_{\mathrm{mc}}^{-1}\left(E_{\perp}, E\right)=2 n d \cdot r_{\perp \mathrm{c}}(E) \sqrt{\frac{E_{\perp}-E_{\perp \mathrm{c}}(E)}{E}} .
$$

The mean path length $\lambda_{\mathrm{mc}}$ which the metachanneled ion travels till the critical collision with atomic row decreases with $E_{\perp}$ as $\left(E_{\perp}-E_{\perp \mathrm{c}}\right)^{-\frac{1}{2}}$.

The equation $\lambda_{\mathrm{mc}}^{-1}\left(E_{\perp}, E\right)=\lambda_{\mathrm{a}}^{-1}(E)$ is readily solved for $E_{\perp \mathrm{mc}}(E)$, the critical transverse energy that provides the equality of stopping rates in a crystal and in an amorphous medium. It is easy to obtain from Eqs. (15) and (14) that 


$$
E_{\perp \mathrm{mc}}(E) \equiv E \psi_{\mathrm{mc}}^{2}(E)=E_{\perp \mathrm{c}}(E)+E \cdot\left[\frac{\pi r_{\perp \mathrm{c}}(E)}{2 d}\right]^{2}
$$

where $\psi_{\mathrm{mc}}(E)=\sqrt{E_{\perp \mathrm{mc}}(E) / E}$ is the critical angle of metachanneling.

Though Eq. (16) is correct at arbitrary ion-atomic interaction potentials, $E_{\perp c}$ and $r_{\perp c}$ are depending of the form of potential. An explicit analytical formula can be obtained in SSA for the standard static Lindhard string potential (11).

For this potential, we have found an exact solution of Eq. (9):

$$
r_{\perp \mathrm{c}}(\epsilon)=\sqrt{2} \cdot a \cdot\left\{\sqrt{\frac{1+3 \epsilon}{3 \epsilon}} \cdot \cos \left[\frac{\pi}{6}+\frac{1}{3} \arcsin \left(\frac{3}{2} \cdot \frac{1-2 \epsilon}{1+3 \epsilon} \cdot \sqrt{\frac{3 \epsilon}{1+3 \epsilon}}\right)\right]-1\right\}^{\frac{1}{2}}
$$

that is valid for arbitrary $\epsilon=\frac{8}{9} \cdot \frac{E}{E^{\prime}}$ and describes the gradual decrease of the critical distance with the increase of ion energy. According to Eq. (17), $r_{\perp c}=a$ at $\epsilon=\frac{1}{8}$, hence only small values of $\epsilon$ belong to the implantation energy region $E \ll E^{\prime}$ where $r_{\perp c}(\epsilon) \approx a \cdot \epsilon^{-\frac{1}{4}} \cdot\left(1-\frac{5}{4} \sqrt{\epsilon}+\frac{9}{16} \epsilon-\ldots\right)$. Substituting Eq. (17) into Eqs. (8) and (16) one gets the following expressions for critical transverse energies at $\epsilon \ll 1$ :

$$
\begin{gathered}
E_{\perp \mathrm{c}}(\epsilon) \cong U_{\mathrm{s}} \cdot \frac{3}{2} \sqrt{\epsilon} \cdot\left(1+\sqrt{\epsilon}+\frac{5}{8} \epsilon\right), \\
E_{\perp \mathrm{mc}}(\epsilon) \cong U_{\mathrm{s}} \cdot \frac{3}{2} \sqrt{\epsilon} \cdot\left[\left(1+\frac{3 \pi^{2}}{16}\right)+\left(1-\frac{15 \pi^{2}}{32}\right) \sqrt{\epsilon}+\left(5+\frac{27 \pi^{2}}{16}\right) \frac{\epsilon}{8}\right] .
\end{gathered}
$$

While Eq. (18) agrees well with the conventional Lindhard's estimate $E_{\perp c} \approx E \psi_{2}^{2}$, Eq. (19) represents a new critical parameter.

The transverse energy range width ratio of the metachanneling and stable channeling modes has the form:

$$
\begin{aligned}
\frac{E_{\perp \mathrm{mc}}(\epsilon)-E_{\perp \mathrm{c}}(\epsilon)}{E_{\perp \mathrm{c}}(\epsilon)} & \cong \frac{3 \pi^{2}}{16}-\frac{21 \pi^{2}}{32} \sqrt{\epsilon}\left(1-\frac{8}{7} \sqrt{\epsilon}\right) \\
& \approx 1.851-\sqrt{\frac{7.307 \times \frac{a_{0}}{d} \times \frac{E}{E_{\mathrm{R}}}}{Z_{1} Z_{2}\left(Z_{1}^{\frac{2}{3}}+Z_{2}^{\frac{2}{3}}\right)}}
\end{aligned}
$$

where $E_{\mathrm{R}}=e^{2} / 2 a_{0}=13.6 \mathrm{eV}$ is the Rydberg energy. One can see that at low energies the range width of metachanneling region can even exceed that of stable channeling. But the ratio decreases as energy increases. It also follows from Eq. (20) that the relative width of the metachanneling mode grows with the increase of the ion and target atomic numbers and of the spatial period $d$ of atomic row. Thus, the significance of dynamically unstable states of directed motion increases with the decrease of ion energy, and is greater for heavier ions and less open channels.

The results of calculations performed for more realistic ZBL potential and channel model agree well with these simple analytical estimations. The B and As ions energy dependencies of $\psi_{\mathrm{c}}(8,9), \psi_{\mathrm{mc}}(16)$ and $\psi_{\mathrm{b}}$ are shown in Fig. 4. These critical angles were calculated by the MICKSER code that accounts for actual geometry of the $\langle 001\rangle \mathrm{Si}$ axial channel (see Sec. IIB), and thus predicts the threshold energies and low-energy maxima of $\psi_{\mathrm{c}}(E)$ and $\psi_{\mathrm{mc}}(E)[22]$. Above these maxima the analytical SSA expressions (18-19) are accurate within $\sim 10 \div 15 \%$. The blocking angle $\psi_{\mathrm{b}}(E)$ has been calculated using the methods of refs. $[12,48]$ for ions emerged from the lattice site position. One can see that $\psi_{\mathrm{mc}}(E)$ is considerably smaller than $\psi_{\mathrm{b}}(E)$. It is due to the fact that $r_{\perp_{\mathrm{c}}}(E)>u_{\mathrm{T}}$ in the implantation energy range, and strong critical collisions occur at rather large distances from the string axis. For the static Lindhard potential, $\psi_{\mathrm{mc}}$ is $\approx 10 \%$ smaller than $\psi_{\mathrm{b}}$ at all energies of interest.

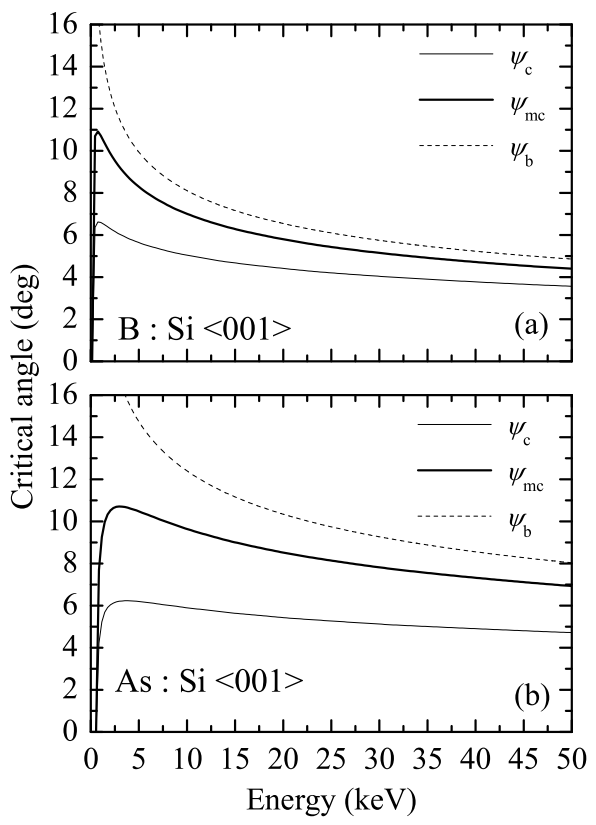

Fig. 4. The energy dependencies of the critical angles of stable channeling $\left(\psi_{\mathrm{c}}\right)$, metachanneling $\left(\psi_{\mathrm{mc}}\right)$, and blocking $\left(\psi_{\mathrm{b}}\right)$ for $\mathrm{B}$ and $\mathrm{As}$ ions in $\langle 001\rangle \mathrm{Si}$ axial channel. 


\section{I. BRATCHENKO, A. S. BAKAI, S. V. DYULDYA}

Predictions of the proposed simple model agree well with the MD modeling results. The solid vertical lines in Fig. 3 indicate the ratio $E_{\perp \mathrm{mc}} / E_{\perp \mathrm{c}}$ for $15 \mathrm{keV} \mathrm{B}$ and $\mathrm{As}$ ions in $\langle 001\rangle \mathrm{Si}$ and testify that just the value of $E_{\perp \mathrm{mc}}$ calculated according to Eq. (16) determines in average the condition of coincidence of $|d E / d x|$ and $|d E / d x|_{\mathrm{a}}$. The right parts of Fig. 3 inset plots demonstrate the calculated contour lines that circumscribe the angular acceptances of the metachanneling in the axial channels adjacent to $\langle 001\rangle$. The comparison with the azimuthal maps of the left parts shows that they adequately characterize the regions where $|d E / d x|<|d E / d x|_{\mathrm{a}}$.

It is seen in Fig. 4 that at $7^{\circ}$ tilt angle standard inci- dence considerable fraction of the low-energy ion beam can be captured into the metachanneling mode immediately on the crystal surface. Besides, the volume capture into this type of motion is feasible because not only slow diffusional but strong ("umklapp") scattering processes can contribute as this mode is not so much blocked as the stable channeling. One can conclude that, because the specific energy losses of the metachanneled ions are depending on $E_{\perp}$, the directional dependence of implantation profile can be observed at any angles of incidence since even at the incidence off the metachanneling angular range ions are free to be captured into this mode in the bulk.
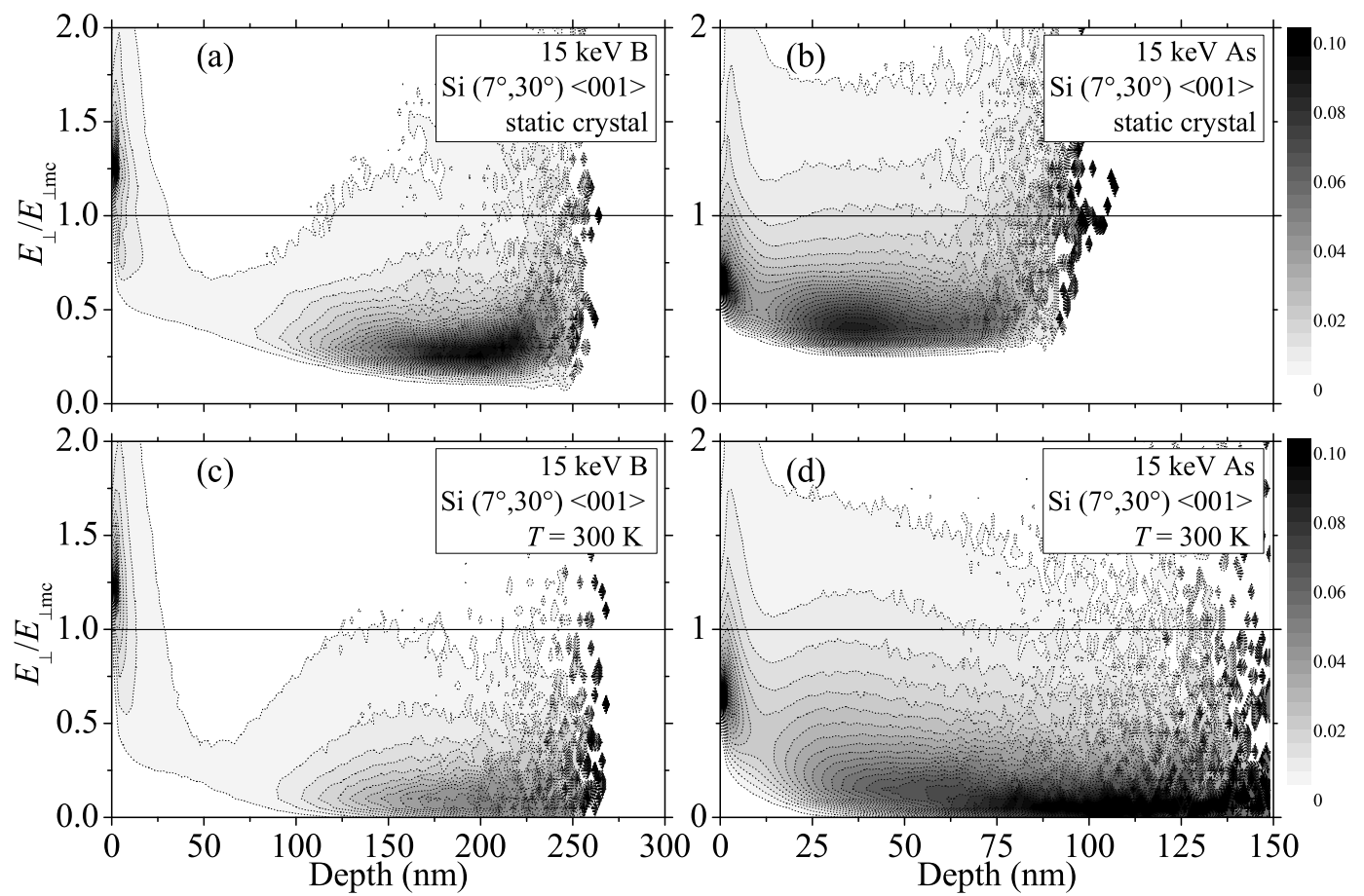

Fig. 5. Normalized distributions of moving implants in the reduced transverse energy $E_{\perp} / E_{\perp \mathrm{mc}}$ as functions of penetration depth for static Si lattice $(\mathrm{a}, \mathrm{b})$ and at $T=300 \mathrm{~K}(\mathrm{c}, \mathrm{d})$. Off-axis $\left(7^{\circ}, 30^{\circ}\right)$ implantation of $15 \mathrm{keV} \mathrm{B}(\mathrm{a}, \mathrm{c})$ and As (b,d) ions.

These considerations are illustrated by Fig. 5. It is similar to Fig. 2, and has been obtained by a similar method except for the fact that transverse energy has been reduced to the current value of $E_{\perp \mathrm{mc}}(E)$, the critical transverse energy (16) of metachanneling. One can see that at $z>R_{\mathrm{p}}$ stationary distributions are formed that keep their shape up to the deep edge of the channeling tail. Along with that, they are practically confined within the range $E_{\perp}<E_{\perp \mathrm{mc}}$ for both ions and both in static and thermally vibrating lattices. One can conclude that the deep tails at off-axis implantation are formed not only due to the volume capture into the stable channeling mode but because of either surface or volume capture into the metachanneling. Therefore, just the critical transverse energy $E_{\perp \mathrm{mc}}(16)$ has to be adopted as the physically based critical parameter that separates the regions of directed and chaotic "random-equivalent" motion.
In Fig. 6 are shown the dechanneling functions $P_{\mathrm{ch}}(z)$ and $P_{\mathrm{mch}}(z)$ defined as the fractions of stably channeled $\left(E_{\perp}<E_{\perp \mathrm{c}}\right)$ and "channeled plus metachanneled" $\left(E_{\perp}<E_{\perp \mathrm{mc}}\right)$ implants, respectively. They correspond to the $\left(E_{\perp}, z\right)$ maps of Figs. 2 and 5, and to the doping profiles of Fig. 1. The roughly exponential decay of $P_{\mathrm{ch}}$ and $P_{\text {mch }}$ at large $z$ is well correlated with the exponential shape of the tails of profiles.

Evidently, $P_{\mathrm{mch}}(z)$ is always larger than $P_{\mathrm{ch}}(z)$ since $E_{\perp \mathrm{mc}}>E_{\perp \mathrm{c}}$ according to Eq. (16). The role of metachanneling is decisive at those $z$ where $P_{\text {mch }}$ and $P_{\text {ch }}$ differ substantially. It takes place at small penetration depths, and in the static case. Namely the metachanneling opens the possibility for B ions to penetrate up to $z \sim 150 \mathrm{~nm}\left(\approx 2.5 R_{\mathrm{p}}\right.$ in $\left.\mathrm{a}-\mathrm{Si}\right)$. At larger depths maximal ranges are reached by stably channeled ions with the transverse energy distribution peaked at $E_{\perp}<E_{\perp c}$.

Thermal vibrations essentially affect the probability 
of the near-surface capture into the stable channeling mode. However, they are not so important as for the trapping into metachanneling. Only $\approx 15 \%$ of light B ions are metachanneled just below the crystal surface. But it means that the surface capture into this mode is not totally prohibited by the blocking effect, as it occurs for the stable channeling.

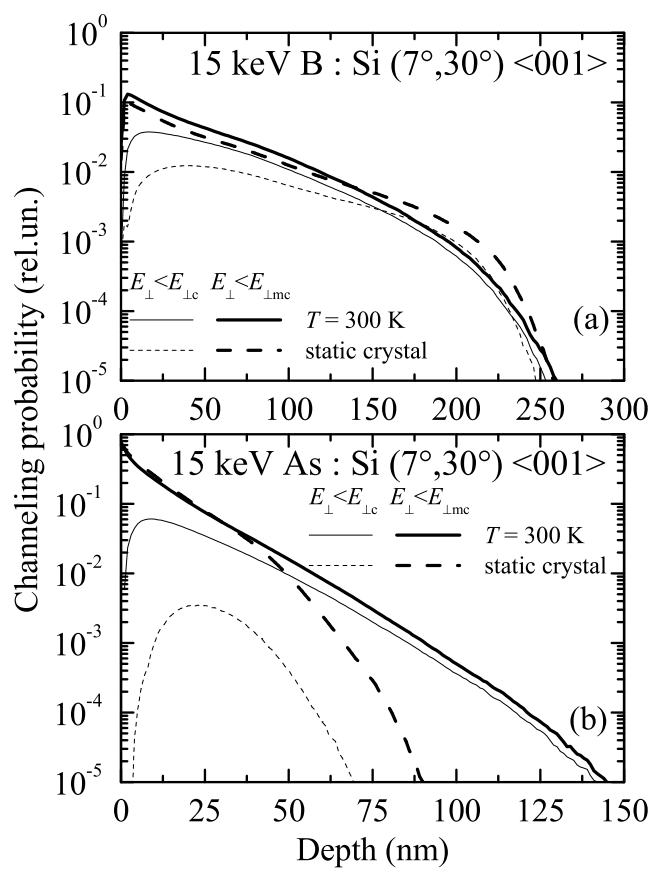

Fig. 6. The MICKSER code calculated depth dependencies of the dechanneling functions $P_{\mathrm{ch}}=\operatorname{prob}\left(E_{\perp}<E_{\perp \mathrm{c}}\right)$ and $P_{\text {mch }}=\operatorname{prob}\left(E_{\perp}<E_{\perp \mathrm{mc}}\right)$ for $15 \mathrm{keV} \mathrm{B} \mathrm{(a)} \mathrm{and} \mathrm{As}$ (b) ions $\left(7^{\circ}, 30^{\circ}\right)$ implantation into $\langle 001\rangle \mathrm{Si}$ (static lattice and $T=300 \mathrm{~K})$.

The metachanneling of heavy As ions is dominant at the surface region since even the surface capture takes place at $7^{\circ}$ tilt incidence. The near-surface capture into the stable channeling is weaker by orders of magnitude, especially in a static crystal. Metachanneling is responsible for the penetration of As implants up to $50 \div 70 \mathrm{~nm}$ (as compared to $16.1 \mathrm{~nm} R_{\mathrm{p}}$ in a-Si). At finite temperatures, it provides considerable diffusional flow of heavy ions toward the stable channeling conditions that are responsible for the maximal length of the channeling tail.

\section{OPTIMIZATION OF REO IMPLANTATION CONDITIONS}

Possibility of practical application of the developed concept of metachanneling is demonstrated by the following computer experiment. Using the MICKSER MD code we have calculated the integral probabilities $P_{\text {tail }}\left(\psi_{\text {in }}, \phi_{\text {in }}\right)$ of ions to get to the channeling tail at very different conditions of incidence onto a (100)Si surface. Initially ions had uniform angular distribution inside a [001] aligned cone having the apex angle $60^{\circ}$. Ions were considered to belong to the tail if their penetration depth exceeds the sum $R_{\mathrm{p}}+\delta R_{\mathrm{p}}$ of range and straggling in a-Si at initial energy $15 \mathrm{keV}$.

The obtained radial maps of $P_{\text {tail }}$ are shown in Fig. 7 for $\mathrm{B}$ and As ions. Manifestation of target crystallography (indicated with Miller indices of major axes and planes) is clearly seen. The greater spreading of axial spots and planar arcs for As is due to larger critical angles of directed motion of heavier ion.

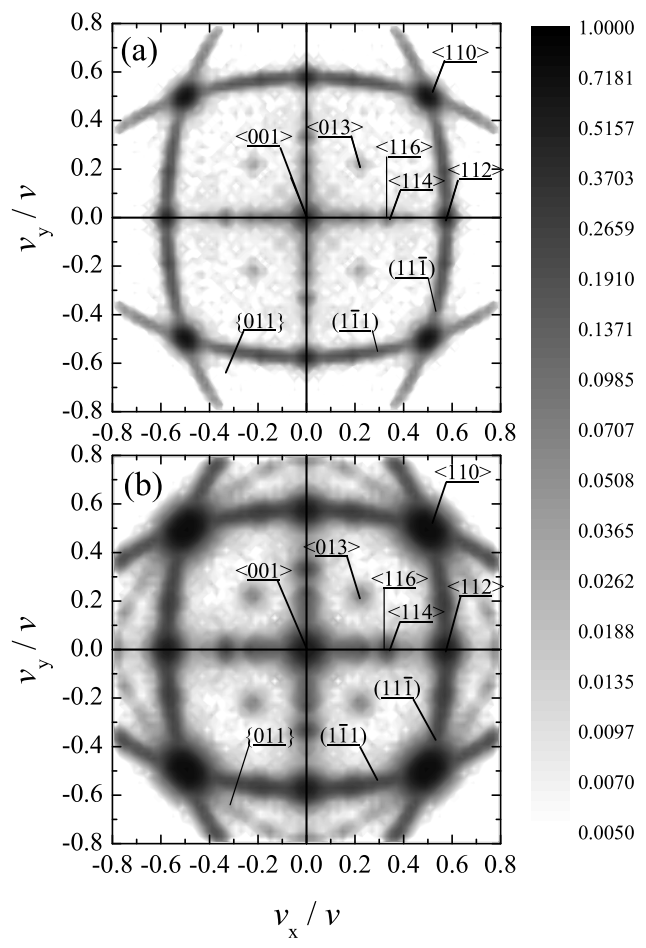

Fig. 7. Radial projections of the probabilities to populate the channeling tail subject to initial conditions of $15 \mathrm{keV} \mathrm{B}$ (a) and As (b) ions incidence onto (100)Si.

Rather important are the results of quantitative analysis of these maps that are illustrated in Fig. 8 by angular cross-sections of of $P_{\text {tail }}\left(\psi_{\text {in }}, \phi_{\text {in }}\right)$ at certain fixed twist angles $\phi_{\text {in }}$. The calculated probabilities were qualified against the critical angles $\psi_{\mathrm{c}}$ and $\psi_{\mathrm{mc}}$ (see vertical arrows in Fig. 8) with respect to all open axial channels ions can be trapped in at given conditions of angular scanning.

The well-defined peaks of the dependencies $P_{\text {tail }}\left(\psi_{\text {in }}, \phi_{\text {in }}=\right.$ const $)$ are formed due to ions captured into the stable channeling in the channels indicated. The rotation angle $\phi_{\text {in }}=30^{\circ}$ can be rated as very close to the optimal one because no effects of adjacent channels are observed near the major [001] axial direction.

The most remarkable feature of Fig. 8 is that the total angular widths of the axial peaks agree well with the quantity $2 \psi_{\mathrm{mc}}\left(E_{\mathrm{in}}\right)$ derived from the critical angles of metachanneling introduced in this paper and indicated in Fig. 8 by solid vertical arrows. This observation is systematically traced for a number of open axial channels resolved. The conventional Lindhard angles $\psi_{\mathrm{c}}\left(E_{\mathrm{in}}\right)$ depicted in Fig. 8(b,e) by dashed arrows have no specific meaning concerning the minimization of $P_{\text {tail }}$. 
Thus, to obtain the optimal (minimal) population of channeling tails, it is necessary and sufficient to misalign the collimated beam off the major axial direction by angle $\psi_{\mathrm{mc}}\left(E_{\mathrm{in}}\right)$. It is smaller than the known blocking an- gle $\psi_{\mathrm{b}}$ but exceeds $\psi_{\mathrm{c}}$ considerably, especially for heavy ions. Reliable estimations can be made with Eqs. (16) and, analytically, (19).

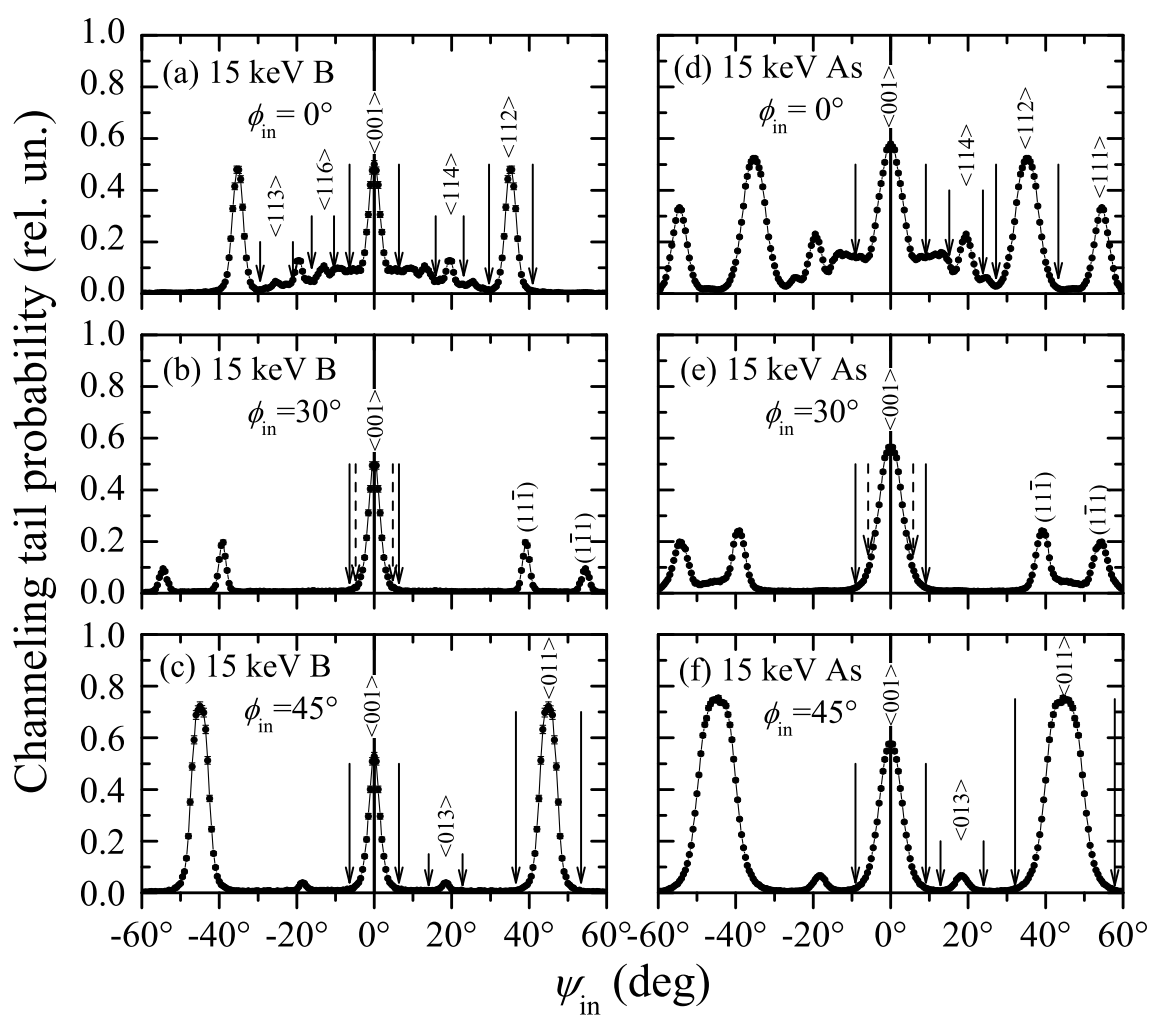

Fig. 8. Angular scans of the probability to populate the channeling tail for $15 \mathrm{keV} \mathrm{B}$ and As ions at different rotation angles $\phi_{\text {in }}$ of Si crystal target.

\section{DISCUSSION AND CONCLUSIONS}

Considerations of the present paper provides a new insight into the microscopic mechanisms of the channeling tails formation at off-axis implantation. At MD simulations, it has been confirmed (Sec. IV) that the existence of long-range tails is intrinsically peculiar to the atomic collisions in crystals. As long as a target is ordered there is no chance to obtain truly random-equivalent doping profiles since the ion stopping in lattices remains directionally dependent. But it is quite possible to suppress the population of tails by careful orientation of the implanter beam with respect to the lattice axes and planes (see Sec. VII). We propose the physically based guideline to identify the optimal alignment conditions.

As was repeatedly demonstrated $[15,18,52]$, the standard $\left(7^{\circ}, 30^{\circ}\right) \mathrm{REO}$ is not optimal generically. For heavy low-energy implants the $\left(\psi_{\mathrm{in}}, \phi_{\mathrm{in}}\right)$ orientation that minimizes the channeling tails is energy and mass dependent. It can be to some extent explained by the known directional effects, quasichanneling and blocking, having the non-trivial characteristic angle $\psi_{\mathrm{b}}(E)$ at low energies. But in practice, very large REO tilt angles have drawbacks such as the effects of neighboring channels, the shadowing of implants by the target mask edges and the enhancement of wafer sputtering that is crucial at high doses. Thus minimal misalignments $\left(\psi_{\text {in }}<\psi_{\mathrm{b}}\right)$ from the target normal are generally favorable.

We have shown (Sec. V-VII) that the actual optimum $\psi_{\text {in }}=\psi_{\mathrm{mc}}(E)$ exists where $\psi_{\mathrm{c}}(E)<\psi_{\mathrm{mc}}(E)<\psi_{\mathrm{b}}(E)$. A reliable theoretical model has been proposed in Sec. VI to evaluate the new critical angle $\psi_{\mathrm{mc}}(E)$ according to Eqs. (16) and (19). It follows from the revealed transient mode of dynamically unstable directed motion of low-energy heavy ions, the metachanneling. Actually, the transition from stable channeling to quasichanneling is not stepwise and takes place in the finite range of abovebarrier $E_{\perp}$. This range becomes larger as the ion energy decreases and the ion mass increases. It is characterized by cooperative effects of the continuum potentials of axial channels and strong collisions having the probability regularly grown with $E_{\perp}$. At metachanneling in ordered lattices, the macroscopic cross-section of strong collisions remains smaller than that in a structureless media. As a result, the stopping is reduced. So, just the metachanneling determines the actual width of the channeling dip of the specific energy losses $|d E / d x|$ of implants. It affects significantly the formation of long-range tails alongside with the known volume capture of ions into the stable channeling that has been widely discussed earlier.

At off-axis implantation of heavy ions, the surface capture into the metachanneling is quite possible in contrast 
to the capture into the stable channeling that is completely due to the scattering on thermal vibrations and effectively occurs from the metachanneled states of motion. Thus the upper bound of the metachanneling rated with the introduced critical angle $\psi_{\mathrm{mc}}(E)$ has to be chosen as an optimal REO condition to minimize the population of the channeling tails, as it has been confirmed by direct computer modeling in Sec. VII.

Finally, one can conclude that the consideration of dynamically unstable above-barrier motion of implants pro- vides the advanced theoretical assessment of the role of lattice driven effects in ion implantation as compared to the conventional treatment based on Lindhard critical angles of stable channeling. It opens up new possibilities of more accurate control of the doping profiles at off-axis implantation. Besides, it has to be taken into account in studies of the spatial structure of collision cascades in crystalline solid, and the induced radiation damage of materials.
[1] Handbook of ion implantation technology, edited by J. F. Ziegler (North-Holland, Amsterdam, 1992).

[2] E. Chason et al., J. Appl. Phys. - Appl. Phys. Rev. 81, 6513 (1997).

[3] International technology roadmap for semiconductors, 2005 edition (2005).

[4] J. F. Ziegler, J. P. Biersack, U. Littmark, The Stopping and Range of Ions in Solids, Stopping and Range of Ions in Matter. Vol. 1 (Pergamon Press, New York, 1985).

[5] http://www.srim.org.

[6] D. S. Gemmell, Rev. Mod. Phys. 46, 129 (1974).

[7] M. A. Kumachov, G. Schirmer, Atomic collisions in crystals (Gordon and Breach, New York, 1989).

[8] R. S. Nelson, M. W. Thompson, Philos. Mag. 8, 1677 (1963).

[9] J. Lindhard, Phys. Lett. 12, 126 (1964).

[10] G. R. Piercy, F. Brown, J. A. Davies, M. McCargo, Phys. Rev. Lett. 10, 399 (1963).

[11] M. T. Robinson, O. S. Oen, Phys. Rev. 132, 2385 (1963).

[12] J. Lindhard, K. Dansk. Vidensk. Selsk. Mat.-fys. Medd. 34, 1 (1965).

[13] M. A. Kumakhov, V. A. Muralev, Phys. Status Solidi A 65, 107 (1981).

[14] S. Mehta, U. Jeong, J. Liu, B. Guo, Mater. Sci. Eng. B 114-115, 72 (2004).

[15] D. R. Myers, R. G. Wilson, J. Comas, J. Vac. Sci. Technol. 16, 1893 (1979).

[16] D. K. Myers, R. E. Wilson, Rad. Eff. 47, 91 (1980).

[17] A. E. Michel et al., Appl. Phys. Lett. 44, 404 (1984).

[18] W. R. Allen et al., Nucl. Instrum. Methods Phys. Res. B 7-8, 265 (1985).

[19] K. Suzuki, K. Ikeda, Y. Yamashita, S.-i. Takagi, Jpn. J. Appl. Phys. 46, 926 (2007).

[20] R. F. Lever, K. W. Brannon, J. Appl. Phys. 69, 6369 (1991).

[21] M. Temkin, I. Chakarov, R. Webb, Nucl. Instrum. Methods Phys. Res. B 164-165, 74 (2000).

[22] V. V. Rozhkov, S. V. Dyul'dya, Sov. Tech. Phys. Lett. 10, 499 (1984).

[23] V. V. Rozhkov, Phys. Status Solidi B 96, 463 (1979).

[24] M. I. Bratchenko, S. V. Dyul'dya, A. S. Bakai, Problems At. Sci. Tech., Ser.: Vacuum, Pure Materials and Superconductors 13, 179 (2006) [in Russian].

[25] M. Posselt, Radiat. Eff. Defects Solids 130-131, 87 (1994).

[26] K. M. Klein, C. Park, A. F. Tasch, IEEE Trans. Elect. Dev. 39, 1614 (1992).

[27] G. Hobler, S. Selberherr, IEEE Trans. on CAD 8, 450
(1989).

[28] S. Tian, J. Appl. Phys. 93, 5893 (2003).

[29] K. Nordlund, Comp. Mat. Sci. 3, 448 (1995).

[30] K. M. Beardmore, N. Grønbech-Jensen, Phys. Rev. E 57, 7278 (1998).

[31] M. I. Bratchenko, S. V. Dyul'dya, V. V. Rozhkov, in $A b-$ str. 5th Rus.-Japan. Int. Symp. on Interact. Fast Charged Part. with Solids (Belgorod, Russia, 1996), p. 21.

[32] S.V. Dyul'dya, M. I. Bratchenko, in Abstr. 5th Conf. High Energy, Nucl. Phys. and Accel. (Kharkiv, Ukraine, 2007), p. 94 [in Russian].

[33] M. I. Bratchenko, S.V. Dyul'dya, in Abstr. 17th Int. Conf. on Phys. of Rad. Phenomena and Rad. Mat. Sci. (Alushta, Ukraine, 2006), p. 63 [in Russian].

[34] M. I. Bratchenko, A. S. Bakai, S.V. Dyul'dya, in Abstr. 5th Conf. High Energy, Nucl. Phys. and Accel. (Kharkiv, Ukraine, 2007), p. 94 [in Russian].

[35] M. I. Bratchenko, A. S. Bakai, S.V. Dyul'dya, in Abstr. 6th Conf. High Energy, Nucl. Phys. and Accel. (Kharkiv, Ukraine, 2008), p. 109 [in Russian].

[36] S. V. Dyul'dya, M. I. Bratchenko, M. A. Skorobogatov, in it Abstr. 6th Conf. High Energy, Nucl. Phys. and Accel. (Kharkiv, Ukraine, 2008), p. 110 [in Russian].

[37] W. Eckstein, Computer Simulation of Ion-Solid Interactions (Springer-Verlag, New York, 1991).

[38] O. B. Firsov, Sov. Phys. JETP 36, 1076 (1959).

[39] L. M. Kishinevskii, Bull. Acad. Sci. USSR, Phys. Ser. 26, 1433 (1962).

[40] D. Cai, N. Grønbech-Jensen, C. M. Snell, K. M. Beardmore, Phys. Rev. B 54, 17147 (1996).

[41] W. Brandt, M. Kitagawa, Phys. Rev. B 25, 5631 (1982).

[42] B. R. Appleton, C. Erginsoy, W. M. Gibson, Phys. Rev. 161, 330 (1967).

[43] M. A. Kumakhov, V. A. Muralev, Izv. Akad. Nauk. SSSR, Ser. Fiz. 37, 2585 (1973) [in Russian].

[44] M. A. Kumakhov, Sov. Phys. Usp. 18, 203 (1975).

[45] L. T. Chadderton, Radiat. Eff. Defects Solids 27, 13 (1975).

[46] E. A. Kovaleva, E. T. Shipatov, Radiat. Eff. Defects Solids 84, 301 (1985).

[47] A. I. Akhiezer et al., Phys. Usp. 38, 1119 (1995).

[48] A. F. Tulinov, Sov. Phys. Dokl. 10, 463 (1965).

[49] G. Buschhorn et al., Phys. Rev. B 55, 6196 (1997).

[50] V. R. Saunders et al., CRYSTAL2003 User's Manual (University of Torino, Torino, 2003).

[51] O. B. Firsov, Radiat. Eff. Defects Solids 21, 265 (1974).

[52] J.-Y. Lee et al., in Proc. 1998 Int. Conf. Ion Implant. Tech. 2, 720 (1999). 


\title{
ВПЛИВ ДИНАМІЧНО НЕСТІЙКОГО КАНАЛЮВАННЯ НА ПОЗАВІСНУ ЙОННУ ІМПЛАНТАЦІЮ
}

\author{
М. І. Братченко*, О. С. Бакай, С. В. Дюльдя \\ Національний науковий центр "Харківсъкий фізико-технічний інститут" \\ вул. Академічна, 1, 61108, Харків, Украӥна, \\ *e-mail: mbrat@kipt.kharkov.ua
}

\begin{abstract}
Орієнтаційні ефекти в імплантації важких йонів у монокристалічні мішені досліджено теоретично та шляхом розгорнутого числового моделювання за допомогою розробленого нового молекулярно-динамічного коду MICKSER з кінцевою метою розв'язання проблеми придушення довогопробіжних хвостів каналювання йонів за умов позавісної імплантації. Уперше виявлено, що частина надбар'єрних йонів зазнає метаканалювання - специфічного режиму динамічно нестійкого орієнтованого руху. Воно є перехідним режимом між стійким каналюванням та квазіканалюванням, забезпечує зменшене гальмування йонів та впливає на імплантаційні профілі легування. Запропонована аналітична модель дала змогу ввести нові критичні поперечну енергію та кут метаканалювання, які визначають верхню межу лунки каналювання енергетичних утрат йонів у низькоенергетичній імплантації. Показано, що мінімізації хвостів каналювання практично можна досягнути за умови, коли кут нахилу мішені дорівнює критичному кутові метаканалювання.
\end{abstract}

